\title{
Regionalentwicklung durch Biokraftstoffproduktion
}

Malawi ist das einzige Land in Afrika, in dem seit vielen Jahren Biokraftstoff in industriellem Maßstab produziert wird. Welche sozio-ökonomischen Auswirkungen hat die zuckerrohrbasierte Ethanolproduktion für Vertragsbauern und Arbeiter? Von Raoul Herrmann und Michael Brüntrup

l: $\mathrm{n}$ der Diskussion um die Expansion der Produktion von Biokraftstoffen (BKS) und ihren sozialen und ökonomischen Auswirkungen nehmen die Länder Subsahara-Afrikas (SSA) eine zentrale Rolle ein, sowohl im positiven wie im negativen Sinne. Dabei geht es insbesondere um den Anbau im industriellen Maßstab. Die BKS-Produktion für den lokalen Verbrauch ist wesentlich weniger umstritten und wird in diesem Text nicht angesprochen.

\section{Industrielle Biokraftstoff-Produktion in Afrika}

Einerseits wird das Produktionspotenzial für BKS zumindest in einigen Ländern S SAs als sehr hoch eingeschätzt. Zahlreiche Länder haben Strategien und Politiken zur Etablierung heimischer BKS-Industrien entwickelt, um dieses Potenzial zu realisieren. Parallel dazu versuchen internationale Investoren in den letzten Jahren, großflächige agro-industrielle Komplexe zur Produktion von BKS in SSA zu entwickeln. Ihre Hauptorientierung ist bisher eher der Export als die lokalen Märkte, denn trotz aller theoretischen Unterstützung durch die Politik für lokale BKS-Nutzung blieb die praktische Umsetzung meist schwach und inkonsistent. Andererseits sind spätestens seit der Nahrungspreiskrise in den Jahren 2007/08 die großflächigen Ansätze zur BKS-Produktion mit agroindustriellen Komplexen äußerst umstritten. Neben grundsätzlichen Bedenken gegen solche Wirtschaftsformen gibt es eine ganze Reihe von befürchteten und beobachteten negativen sozio-ökonomischen und politischen Auswirkungen. Dazu gehören Enteignungen, Verdrängung des Nahrungsanbaus, Abhängigkeiten und nicht zuletzt ökologische Schäden.

Die möglichen positiven und negativen Wirkungen großer B KS-Komplexe sind jeweils plausibel, aber in ihrer Gesamtheit und empirisch noch nicht gut verstanden. Erschwerende Faktoren sind, dass die Wirkungen jeweils von den lokalen Bedingungen und der Vertragsgestaltung abhängen; dass eine Vielzahl von Betroffenen zu berücksichtigen ist; und dass einzelne Wirkungen sehr stark vom Zeitpunkt abhängen, wobei zu- nächst hauptsächlich Kosten und erst später, wenn überhaupt, Erträge zu erwarten sind.

Empirisch gut belegt sind viele negative Wirkungen von großen Landakquisitionen der letzten Jahre. Allerdings gibt es viel weniger empirische Untersuchungen zu den langfristigen Wirkungen von Investitionen auf solchen Ländereien. Zum einen handelt es sich um ein junges Phänomen, und es dauert Jahre und Jahrzehnte von der Landakquisition bis zur vollen Produktion.

\section{Bioethanol aus Malawi}

Malawi bietet eine der wenigen Möglichkeiten, die längerfristigen Auswirkungen der industriellen BKS-Produktion und -Nutzung in SSA zu analysieren. In den 1980er Jahren haben einige andere Länder nach den Ölpreiskrisen in den 1980er Jahren mit der Produktion von Bioethanol begonnen. Der bekannteste Fall ist Brasilien; in SSA waren es beispielsweise Simbabwe, Sambia, Kenia und Südafrika. Zwischenzeitlich haben die meisten die Produktion wieder eingestellt. Malawi ist das einzige Land in SSA, das seither kontinuierlich im großen, industriellen Maßstab BKS erzeugt.

Bioethanol wird in Malawi bisher aus Melasse, einem Nebenprodukt der Zuckerproduktion, erzeugt. Die BKS-Produktion wird begünstigt durch eine hohe Produktivität der Zuckerrohrproduktion mit Bewässerung, und durch den Mangel an Verwertungsmöglichkeiten für die Melasse. Als Binnenland muss Malawi alle Importe durch Transitländer und mit hohen Transportkosten beziehen. Die Kraftstoffkonzerne dürfen bis zu 20 Prozent Bioethanol beimischen. Sie müssen die entsprechende Menge zu einem knapp unter dem Importpreis für Benzin liegenden Preis aufkaufen.

Die sozio-ökonomischen Auswirkungen der BKS-Produktion entscheiden sich hauptsächlich im primären Anbau, weniger in der Weiterverarbeitung. Sie hängen insbesondere vom Geschäftsmodell der Rohstoffproduktion ab, das heißt von Technologie, Akteuren und Geschäftsbeziehungen. In Malawi ist die Zuckerproduktion hauptsächlich in der Hand eines einzigen ausländischen Konzerns, der die Melasse an eine inländische Firma verkauft, die sie weiterverarbeitet. Die älteren Zuckerrohr-Anbauflächen sind großflächige Plantagen im Eigentum der Zuckergesellschaft, die mit einer hohen Zahl an Arbeitern teilmechanisiert bewirtschaftet werden. Die jüngeren Expansionen wurden in erster Linie im Rahmen von Vertragsanbau von Kleinbauern realisiert, wobei diesen eine garantierte Marktnachfrage sowie Zugang zu Krediten, landwirtschaft- 


\section{„Verbesserte Einkommen der Kleinbauern reichen nicht für eine nachhaltige Produktion."}

licher Beratung und Inputs, vor allem Mineraldünger, zugesichert wird. Der kleinbäuerliche Anbau erfolgt zum Teil geografisch konzentriert im Rahmen von großen beweglichen Rundbewässerungsanlagen. Aufbau der Bewässerungsanlagen inklusive der notwendigen Bodenumverteilung wird von einer staatlichen Agentur organisiert und vorfinanziert, die Organisation der Zuckerrohrproduktion und des Transportes zur Fabrik erfolgt durch früher staatliche, jetzt privatisierte Managementfirmen.

Eine Studie an einem der beiden Standorte zeigt, dass in dieser Region, die traditionell dominiert wird von subsistenzorientiertem Nahrungsmittelanbau und semikommerziellem Reisanbau ohne Bewässerung, durch die Einführung der intensiven Zuckerrohrproduktion substanzielle Verbesserungen der wirtschaftlichen Verhältnisse von teilnehmenden Bauern und Arbeitern erzielt werden können (Herrmann et al. 2013). Insbesondere Vertragsbauern haben mehrfach erhöhte Einkommen und konnten Kapitalgüter akkumulieren. Weitere Analysen zeigen, dass sich die Befürchtungen negativer Auswirkung des Zuckerrohranbaus auf die Nahrungsmittelproduktion der Haushalte nicht bewahrheitet haben. Gründe hierfür sind, dass Abnahmeverträge explizit auch Kredite für Maissaatgut und -dünger beinhalten, dass die Zuckerrohr-Abnahmeverträge bei der Bank als Sicherheit für weitere Kredite hinterlegt werden können und dass das steigende Bareinkommen den Bauern erlaubt, Ersparnisse aufzubauen.

Der untersuchte Fall zeigt zudem, dass die Beschäftigung auf Plantagen in der Regel besser entlohnt wird als alternative Lohnarbeit im ländlichen Raum. Ein Grund dürfte sein, dass ein internationales Unternehmen involviert ist, das stärker unter Kontrolle durch internationale und nationale Agenturen steht und höhere Standards einhalten muss. Außerdem ist die Produktivität auf diesen Betrieben hoch, was höhere Löhne ermöglicht. Hingegen ist der kleinbäuerliche Sektor in diesen Regionen wenig entwickelt, und außerlandwirtschaftliche Arbeitsplätze sind rar und schlecht bezahlt. Die Nachfrage nach Arbeitsplätzen auf den Plantagen ist entsprechend hoch und wird es wohl so lange bleiben, bis sich genügend Alternativen aufgetan haben.

Jedoch zeigt der Fall Malawi auch, dass eine verbesserte Einkommenslage der Beteiligten alleine nicht ausreicht, um eine industrielle BKS-Produktion sozial-ökonomisch nachhaltig zu gestalten. So führte die Expansion der Vertragsbauern-Modelle unter zentraler Bewässerung zur Enteignung von vorhergehenden Landnutzern durch die Agentur beziehungsweise lokale Autoritäten, ohne ausreichende Kompensation. Weitere Konflikte entstanden aufgrund von Verteilungsfragen innerhalb der Wertschöpfungskette, da die privatisierten Managementfirmen der Misswirtschaft bezichtigt wurden. Viele betroffenen Bauern fühlten sich übervorteilt und es kam zu gewaltsamen Protesten mit Toten. Die Europäische Union, die bis dahin wesentlich an der Finanzierung der Erweiterung des Vertragsanbaus beteiligt gewesen war, stellte daraufhin finanzielle Zusagen für das Projekt ein und beauftragte eine Kommission mit der Untersuchung der Konflikte. Gleichzeitig erfolgte eine Restrukturierung und Diversifizierung der Managementfirmen, um die Misswirtschaft zu beenden und die Kostenmargen zu reduzieren.

\section{Schlussfolgerungen}

Die Entwicklung einer BKS-Industrie in SSA bietet sozioökonomische Chancen für die Wirtschaft und ländliche Räume, in denen die Rohstoffe hergestellt werden, aber auch viele Risiken. Das Beispiel Malawi ist einer der wenigen Fälle, an denen sich die langfristigen Auswirkungen des Aufbaus eines industriellen BKS-Sektors in SSA zeigen lassen. Die Ergebnisse zeigen, dass positive Wohlfahrtseffekte für beteiligte Kleinbauern und Plantagenarbeiter möglich sind. Diese Gruppen sind für die entwicklungspolitische Bewertung der industriellen B KS-Produktion in SSA zentral. Die agro-industrielle B KS-Produktion in SSA muss also nicht im Widerspruch zu Armutsbekämpfung und Ernährungssicherheit stehen.

Nichtsdestotrotz zeigt der Fall auch, dass Risiken real sind. So entstanden trotz der wirtschaftlichen Vorteile Konflikte, weil es versäumt wurde, vorhergehende Landnutzungsansprüche zu dokumentieren und die Expansionen in einem Bottom-upAnsatz entsprechend den lokalen Bedingungen transparent und fair zu gestalten. Dies erinnert an die vielen Berichte zu Landgrabbing im Umkreis von BKS-Investitionsprojekten. Es bestätigt, dass diese Investitionsphase besonders hohe Risiken und Konfliktpotenziale birgt.

\section{Literatur}

Herrmann, R./Grote, U./Brüntrup, M. (2013): Household Welfare Outcomes of Large-Scale Agricultural Investments: Insights From Sugarcane Outgrower Schemes and Estate Employment in Malawi. In: Annual World Bank Conference on Land and Poverty 2013, Washington D. C., April 8-11, 2013.

\section{AUTOREN + KONTAKT}

Dr. Michael Brüntrup und Raoul Herrmann sind wissenschaftliche Mitarbeiter in der Abteilung Wettbewerbsfähigkeit und soziale Entwicklung des Deutschen Instituts für Entwicklungspolitik (DIE).

Deutsches Institut für Entwicklungspolitik (DIE), Tulpenfeld 6, 53113 Bonn. Tel.: +49 228 94927-267, E-Mail: michael.bruentrup@die-gdi.de, raoul.herrmann@die-gdi.de, Internet: www.die-gdi.de
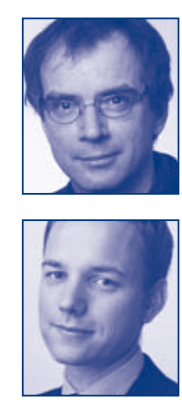
Copyright (C) 2013, IÖW und oekom Verlag. Die Nutzung des Artikels ist Abonnenten von Ökologisches Wirtschaften vorbehalten. Nachdruck und Vervielfältigung des Artikels einschließlich Speicherung und Nutzung auf optischen und elektronischen Datenträgern nur mit Zustimmung der Redaktion von Ökologisches Wirtschaften (http://www.oekologischeswirtschaften.de). 